
\title{
Reseracth Sulure \\ No DC superheating and increased surface pinning in low temperature baked niobium
}

Daniel Andrew Turner ( $\nabla$ daniel.turner@cockcroft.ac.uk)

Lancaster University

Graeme Burt

Lancaster University

\section{Tobias Junginger}

TRIUMF

\section{Research Article}

Keywords: superconducting radio frequency (SRF), niobium

Posted Date: October 1st, 2021

DOI: https://doi.org/10.21203/rs.3.rs-948318/v1

License: (1) This work is licensed under a Creative Commons Attribution 4.0 International License.

Read Full License 


\title{
No DC superheating and increased surface pinning in low temperature baked niobium
}

\author{
Daniel Andrew Turner ${ }^{1,2, *}$, Graeme Burt ${ }^{1,2}$, and Tobias Junginger ${ }^{3}$ \\ ${ }^{1}$ Cockcroft institute, STFC Daresbury Laboratory, Warrington, WA4 4AD, UK \\ ${ }^{2}$ Lancaster University, Engineering, Lancaster, LA1 4YW, UK \\ ${ }^{3}$ TRIUMF, Accelerator division, BC, Canada \\ *daniel.turner@cockcroft.ac.uk
}

\begin{abstract}
Superconducting Radio-Frequency cavities are currently made out of niobium. Niobium cavities are limited by the magnetic field on the cavity walls due to the entry of vortices at the field of first vortex penetration, $\mathrm{H}_{v p}$. Low temperature baking in vacuum or low pressure gas atmosphere removes the strong decrease of the quality factor with accelerating gradient (high field Q-slope). Some cavities reach surface magnetic field above the lower critical field $\mathrm{H}_{c 1}$. One hypothesis for this performance increase is that the outer layer affected by the treatments acts as a barrier for vortex penetration (effective bilayer). Using a vibrating sample magnetometer the field of first flux penetration $\left(\mathrm{H}_{v p}\right)$ was measured for $\mathrm{Nb}$ ellipsoids with various low temperature treatments. All $\mathrm{H}_{v p}$ values were found to be consistent with the lower critical field, $\mathrm{H}_{c 1}$, as predicted for clean niobium. This led to the conclusion that a metastable flux free state above $\mathrm{H}_{c 1}$ cannot be observed in DC magnetometry for low temperature baked niobium unlike for bilayers consisting of two superconductors as previously published. The effect of flux pinning differed significantly between treatments, suggesting that the high field Q-slope mitigation might be related to vortex pinning in the surface of the cavities.
\end{abstract}

\section{Introduction}

Particle accelerators often use superconducting radio frequency (SRF) cavities to accelerate the particle beam. Large electromagnetic fields are produced when the RF power is applied close to the resonant frequency of the cavity. The electric field generated accelerates the particle bunches as they pass through the cavity. The operating frequencies for SRF cavities typically range between $350-3900 \mathrm{MHz}^{1}$ for elliptical multi-cell structures, with the optimum frequency being dependant on the application and the structure of the cavity. As a consequence of Maxwell's equations a magnetic field is generated, which is proportional to the applied electric field. The magnetic field interacts with the cavity surface and is cancelled inside the superconductor by screening currents formed in the surface layer of the superconductor. The maximum accelerating gradient $\left(\mathrm{E}_{a c c}\right)$ is limited by the magnetic field on the cavity walls. Superconductors experience field transitions at critical magnetic fields, which is a response to an externally applied field $\left(\mathrm{H}_{e x t}\right)$. Below the lower critical field $\left(\mathrm{H}_{c 1}\right)$ a type II superconductor will be in the Meissner state and behave as a perfect diamagnet. The field will be expelled from the superconductor due to screening currents which oppose the $\mathrm{H}_{e x t}$ such that $\mathrm{H}_{e x t}$ decays by 1/e over the London penetration depth $\left(\lambda_{L}\right)$. Above $\mathrm{H}_{c 1}$ it becomes energetically favourable for vortices to be present within the superconductor. Currently, accelerating cavities are made out of bulk $\mathrm{Nb}$ due to having the largest critical temperature $\left(\mathrm{T}_{c}\right)$ of any element ${ }^{1}$ and the largest $\mathrm{H}_{c 1}$ for any known superconductor. For clean $\mathrm{Nb}, \mathrm{T}_{c}=9.25 \mathrm{~K}$ and $\mu_{0} \mathrm{H}_{c 1}$ is approximately $174 \mathrm{mT}$ at $0 \mathrm{~K}^{2,3}$. It is possible for a superconductor to remain in the Meissner state above $\mathrm{H}_{c 1}$ up to a superheating field $\left(\mathrm{H}_{s h}\right)$ due to the the Bean-Livingston surface barrier ${ }^{4}$. To reach $\mathrm{H}_{s h}$ the superconducting surface needs to have no imperfections such as dislocation clusters and impurities large enough to act as seeds for flux penetration. Using the Ginzburg-Landau parameter, $\kappa_{G L}$, a relation between $\mathrm{H}_{s h}$ and the thermodynamic critical field $\left(\mathrm{H}_{c}\right)$ can be made. The Ginzburg-Landau parameter is given by $\kappa_{G L}=\lambda_{L} / \xi^{1}$, where for clean $\mathrm{Nb} \kappa_{G L} \approx 1$ and $H_{s h} \approx 1.2 H_{c}$. Therefore, for $\mathrm{Nb}$ with $\mu_{0} \mathrm{H}_{c} \approx 199.3 \pm 10 \mathrm{mT}$ at $0 \mathrm{~K}^{3}, H_{s h}$ can persist up to $\approx 240 m T$ at $0 \mathrm{~K}$. More detailed calculations within Ginsburg-Landau theory can be found in ${ }^{5}$.

To reach large accelerating gradients it is standard practice to perform a low temperature bake (LTB) as a final preparation step. A LTB consists of heating the cavity to $120^{\circ} \mathrm{C}$ for 48 hours in ultra-high vacuum ${ }^{6}$. Recently a modified two-step baking process where the cavity is initially baked at a reduced temperature of $75 \mathrm{C}$ for 4 hours has shown to yield even larger accelerating gradients ${ }^{7}$. Another LTB method which yields accelerating gradients and quality factors in excess to what can be obtained with LTB at $120^{\circ} \mathrm{C}$ is called low temperature nitrogen infusion often simply referred to as nitrogen infusion. Nitrogen infusion is performed by heating the cavity to $800^{\circ} \mathrm{C}$ in high vacuum for 3 hours for $\mathrm{H}$ degassing and to separate any $\mathrm{Nb}_{2} \mathrm{O}$, after which the cavity is cooled and held between $120-200^{\circ} \mathrm{C}$ with a pressure of $25 \mathrm{mTorr}$ of $\mathrm{N}^{8}$. The best $\mathrm{Nb}$ cavities prepared 
by LTB reach a maximum $\mathrm{E}_{a c c}$ of around $50 \mathrm{MV} / \mathrm{m}$ whilst operating at $2 \mathrm{~K}$, which corresponds to a maximum surface magentic field of $\approx 200 \mathrm{mT}^{9}$. This is above $\mu_{0} \mathrm{H}_{c 1}$ for $\mathrm{Nb}$ at $2 \mathrm{~K}$ and approximately $10 \%$ lower than the the $\mathrm{H}_{s h}$ which is the expected maximum value.

It is known that all LTB processes described above yield a larger penetration depth and therefore a reduced screening current in the outer layer exposed to the RF field. Low energy muon spin rotation results have shown that there is a strong change in Meissner screening at a depth of about $60 \mathrm{~nm}$ for $120^{\circ} \mathrm{C}$ baked niobium ${ }^{10}$. This could yield a superconductor-superconductor (SS) barrier for flux penetration at the boundary similar to the Bean-Livingston barrier at the superconductor-vacuum interface ${ }^{11}$ delaying flux penetration and therefore increasing the field of first vortex penetration $\left(H_{v p}\right)$. Assuming time scales are not relevant an increase in $H_{v p}$ would be observable in a DC experiment, where $H_{v p}$ can be measured without the effect of RF heating.

\section{Sample Testing}

The aim of this study is to test with DC magnetometry whether the increase in accelerating gradient caused by different LTB processes can be correlated to an increased DC field of first vortex penetration. High temperature annealed ellipsoidal samples were used to avoid edge and pinning effects. For details on the preparation see the method section. Four samples were tested. One received no further heat treatment after annealing, whilst the other three were subject to a LTB. The samples were tested in a SQUID magnetometer, specifically a Quantum Design MPMS 3. The field applied by a SQUID magnetometer is from a solenoid much larger than the sample itself, and the sample is positioned inside the solenoid such that the applied field is uniform. With an ellipsoidal sample, the flux lines around the ellipsoid will be denser around the equator of the ellipsoid, and therefore the local field on the sample surface is larger than $\mathrm{H}_{e x t}$. The demagnetization factor $N$ relates the field at the equator $H_{e q}$ to $H_{e x t}$ by $H_{e q}=H_{e x t} /(1-N)^{12}$, where $\mathrm{N}=0.13$ for the ellipsoidal samples used in this study.

Generally samples were zero field cooled (ZFC) for each 5 quadrant hysteresis loop measurement at fixed temperature. The reported $\mathrm{H}_{\text {ext }}$ is determined by the current known to be passing through the solenoid which applies the field, and the applied field could be different due to the history of the magnet as flux could be trapped within the solenoid ${ }^{13}$. Therefore after each hysteresis run the magnet was de-gaussed to reduce pinning in the magnet, before the sample was heated above $\mathrm{T}_{c}$ to remove any pinning from the sample. The samples were then warmed up, and held at $12 \mathrm{~K}$ for 5 minutes to expel any flux that could be trapped within the sample, before undergoing ZFC again. As the external field $\mathrm{H}_{\text {ext }}$ is swept it does not stabilise to a specific value so the reported $\mathrm{H}_{\text {ext }}$ are averages ${ }^{13}$.

Each testing cycle begins at $\mu_{0} \mathrm{H}_{\text {ext }}=0 \mathrm{mT}$, such that there is no magnetic moment produced. The external field is then slowly increased which results in a perfect diamagnetic response produced from the superconductor, which is shown in Figure 1 by the initial curve (straight line in fourth quadrant starting from the origin). When the local field on the surface of the superconductor reaches $\mathrm{H}_{v p}$ the flux enters the superconductor dividing the ellipse into normal conducting/superconducting regions. Once the vortices have entered the sample, the superconductor has transitioned from the Meissner state to the Abrikosov state and the response of the magnetic moment to the applied field is no longer linear. This is due to more vortices penetrating into the superconductor, in turn reducing the superconducting volume. As $\mathrm{H}_{\text {ext }}$ continues to be increased, the moment increases until $\mathrm{H}_{c 2}$, where the moment becomes slightly positive due to the paramagnetic response of the normal conducting Nb. The external field is then decreased. By decreasing the field, the flux is then expelled from the superconductor, and the magnetic moment becomes negative again. In the case of a perfect superconductor, the produced magnetic moment would be the same for both increasing and decreasing $\mathrm{H}_{\text {ext }}$. It can be seen in Figure 1 that this is not the case here. The absolute value of the magnetic moment is smaller than for the initial curve. This is due to trapped flux within the sample. After $\mathrm{H}_{\text {ext }}$ has reached zero again the field is ramped at a faster rate with reversed polarity (negative applied field). These results are shown by the black and red curves in Figure 1. Each hysteresis cycle ends with a repetition of the initial virgin curve. This is also done to ensure that the sample has not moved during the test.

\section{Determining the field of first flux penetration}

To determine the field of first flux penetration, only the initial curve produced by increasing $\mathrm{H}_{\text {ext }}$ is used as there is no magnetic history which can affect the results. Whilst in the Meissner state the response of the superconductor is linear due to $\mathrm{H}_{\text {ext }}$, and can be described as $M=K^{*} H_{e x t}{ }^{14}$, where $\mathrm{M}$ is the magnetic moment and $K^{*}$ is a constant proportional to the superconducting volume, which can vary slightly between samples. By normalising $M K^{*} / H$ in the Meissner state to 1 as shown in the bottom left quadrant of Figure 1, $\mathrm{H}_{v p}$ can be determined by the last point to be within error of 1 . Once $\mathrm{H}_{\text {ext }}$ has been found, the geometry of the sample must be taken into account. Due to the geometry of the ellipsoid, $\mathrm{N}$ is 0.13 , such that $H_{v p}=0.87 H_{e x t}$. This method is done for each sample at each temperature.

\section{Determining irreversible pinning strength}

In an ideal pin-free superconductor once $\mathrm{H}_{e x t}$ has increased above $\mathrm{H}_{c 2}$ and is then decreased, the magnetic moment produced by the sample is identical to the initial magnetisation loop. If the superconductor is not pin-free, the return loop for the 


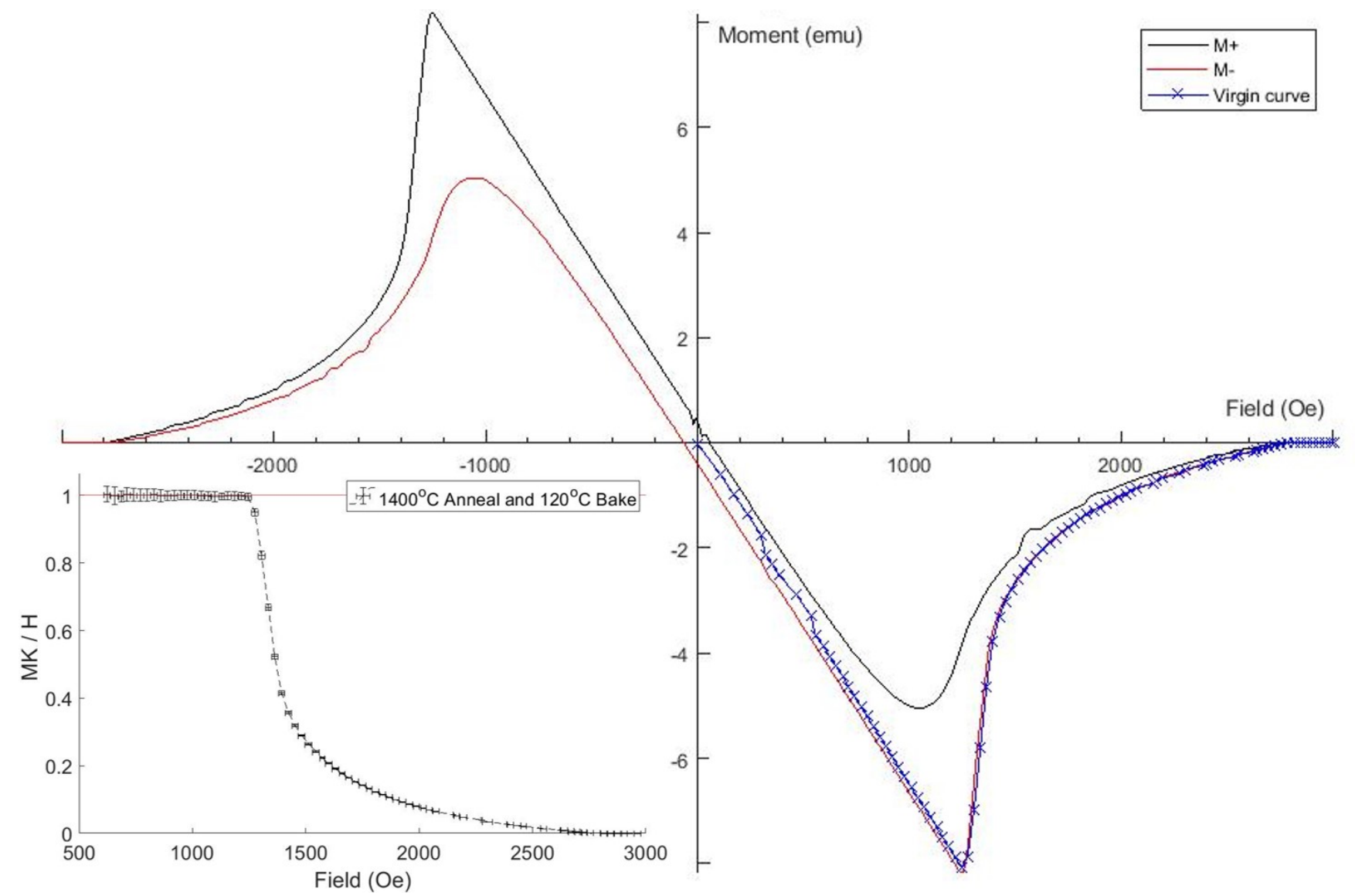

Figure 1. Hysteresis loop for the $120^{\circ} \mathrm{C}$ baked ellipsoid. The initial increase and decrease in the externally applied field is known as the virgin curve, shown in blue. The positive and negative moment used to determine pinning strength are shown in red and black. The standardisation curve used to determine $\mathrm{H}_{v p}$ is shown in the bottom left quadrant, which is determined using the virgin curve. The last point within error of 1 is taken as $\mathrm{H}_{v p}$.

magnetisation curve will differ, which is found in all the hysteresis graphs presented in this paper. To determine the pinning strength produced by each treatment the irreversible magnetization was calculated using the hysteresis loops shown in Figure 1, using both the positive and negative moment. The irreversible magnetization is then found using $M_{i r}=\left(M^{+}-M^{-}\right) / 2^{15}$, with both $\mathrm{M}^{+}$and $\mathrm{M}^{-}$shown in Figure 1. The $\mathrm{M}_{i r}$ is plotted as a function of $\mathrm{H}_{e x t}$ for each sample at 4.2 $\mathrm{K}$ in Figure 5. The $\mathrm{M}_{i r}$ is the largest at $\mathrm{H}_{v p}$ where the return loop does not follow the initial curve due to pinning within the sample. The pinning strength $\left(\mathrm{M}_{p i n}\right)$ for each temperature and treatment is then taken at the point where $\mu_{0} \mathrm{H}_{e x t}$ is $0 \mathrm{mT}$, ie $\mathrm{M}_{i r}(0 \mathrm{Oe})=\mathrm{M}_{\text {pin }}$, as the magnetic moment is being produced by the sample is not a response to $\mathrm{H}_{\text {ext }}$. The irreversible pinning for each treatment is shown in Table 2.

\section{Results}

A hysteresis loop was performed at 2, 3, 4.2, 5, 6, 7, 8, and $9 \mathrm{~K}$ for all samples except the $120^{\circ} \mathrm{C}$ baked one. The effect of temperature on the hysteresis loops can be seen in Figure 2, where the increasing temperature reduces the critical fields of the superconductors. It should be noted that the hysteresis loops for the Baseline, $120^{\circ} \mathrm{C}$ bake and the $75 / 120^{\circ} \mathrm{C}$ bake have similar looking hysteresis curves across all temperatures respectively. I.e. each sample experiences smooth transitions as $\mathrm{H}_{e x t}$ varies. This is not the case for the $\mathrm{N}$ infused sample. It can be seen that after the sample had been increased above $\mathrm{H}_{c 2}$, the moment has some sharp transitions shown in the top left quadrant and the bottom right quadrant (indicated by the arrows) and in low $\mathrm{H}_{\text {ext }}$ shown in Figure 2. These flux jumps are only visible at $2 \mathrm{~K}$. These sharp transitions indicate flux jumps where trapped flux suddenly moves within the sample, from one pinning center to another due to a change of forces as $\mathrm{H}_{e x t}$ is increased and more vortices enter the ellipse. This only happens after the ellipse had already been taken to $\mathrm{H}_{c 2}$ to take the superconductor into the normal conducting regime. There is no flux jump at $2 \mathrm{~K}$ when $\mathrm{H}_{\text {ext }}$ is initially increased and decreased which allows us to determine that the flux has been trapped after the sample was in the normal conducting state with $\mathrm{H}_{e x t}>\mathrm{H}_{c 2}$. 


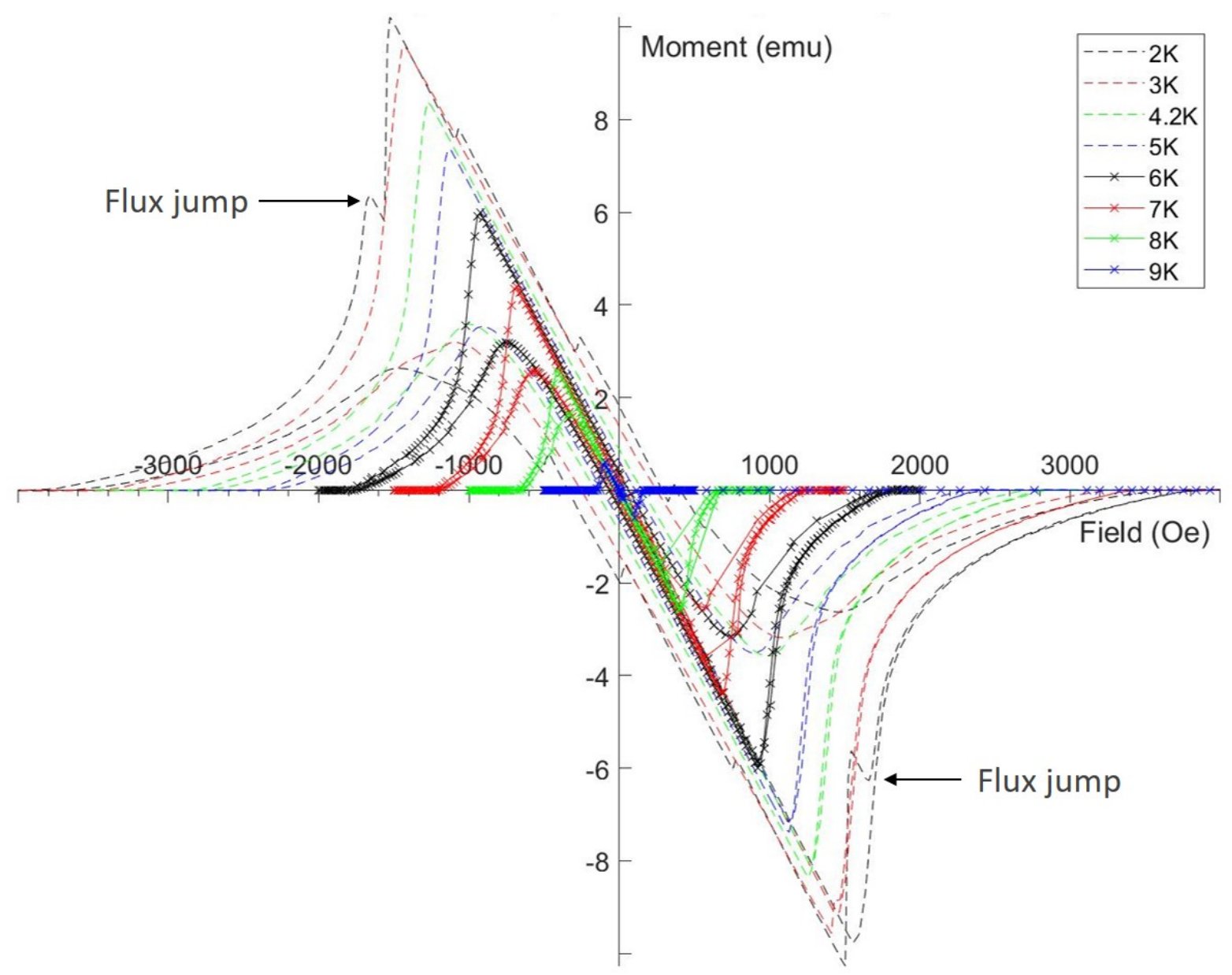

Figure 2. The hysteresis loops performed on the $\mathrm{N}$ infused sample at varying temperatures. Flux jumps can be seen once the sample had been taken above $\mathrm{H}_{c 2}$ for the $2 \mathrm{~K}$ data only.

The field of first vortex penetration was found for each sample at various temperatures by using the standardisation curve method described above. Once $\mathrm{H}_{v p}$ was found and the field enhancement accounted for by the demagnetization factor, a graph of $\mathrm{H}_{v p}$ as a function of temperature could be plotted, shown in Figure 3. It was found that $\mathrm{H}_{v p}$ fits the expression $H_{v p}(T)=H_{v p}(0)\left(1-\left(T / T_{c}\right)^{2}\right)$ allowing extrapolation to determine $\mathrm{H}_{v p}$ at $0 \mathrm{~K}$, as well as extrapolating to $\mathrm{T}_{c}$ when $\mathrm{H}_{v p}=0$ $\mathrm{mT}$. It can be seen from Figure 3 and Table 1 that there is no significant change in $\mathrm{H}_{v p}$ produced by low temperature baking or $\mathrm{N}$-infusion when tested in DC magnetometry. In addition it can be seen in Table 1 that there is no change between extrapolated critical temperature between samples.

\begin{tabular}{|c|c|c|c|c|}
\hline & \multicolumn{4}{|c|}{$\mu_{0} \mathbf{H}_{v p}(\mathbf{T}), \mathbf{m T}$ for each treatment } \\
\hline T, K & Baseline & $\mathbf{1 2 0}^{\circ} \mathbf{C}$ bake & $\mathbf{7 5 / 1 2 0} \mathbf{C}^{\circ}$ bake & N infusion \\
\hline 2 & $174.6 \pm 2.19$ & - & $175.5 \pm 1.21$ & $179.0 \pm 2.06$ \\
\hline 3 & $160.5 \pm 1.84$ & - & $159.9 \pm 1.09$ & $163.7 \pm 2.07$ \\
\hline 4 & $140.7 \pm 2.64$ & $143.1 \pm 1.55$ & $143.4 \pm 2.30$ & $144.7 \pm 2.18$ \\
\hline 5 & $126.3 \pm 2.41$ & - & $127.7 \pm 2.18$ & $129.9 \pm 2.07$ \\
\hline 6 & $104.0 \pm 2.18$ & - & $104.7 \pm 2.19$ & $106.16 \pm 2.07$ \\
\hline 7 & - & - & $78.0 \pm 1.09$ & - \\
\hline $\mathbf{T}_{c}(\mathbf{0} \mathbf{~ m T})$ & $9.362 \pm 0.0142$ & - & $9.360 \pm 0.0118$ & $9.371 \pm 0.0143$ \\
\hline
\end{tabular}

Table 1. The field of full flux penetration for each set temperature and the critical temperature determined by using the linear dependence of $\mathrm{H}_{v p}$ vs $\mathrm{T}^{2}\left(\mathrm{~T}_{c}(0 \mathrm{mT})\right)$, assuming a linear $\mathrm{T}^{2}$ dependence. 


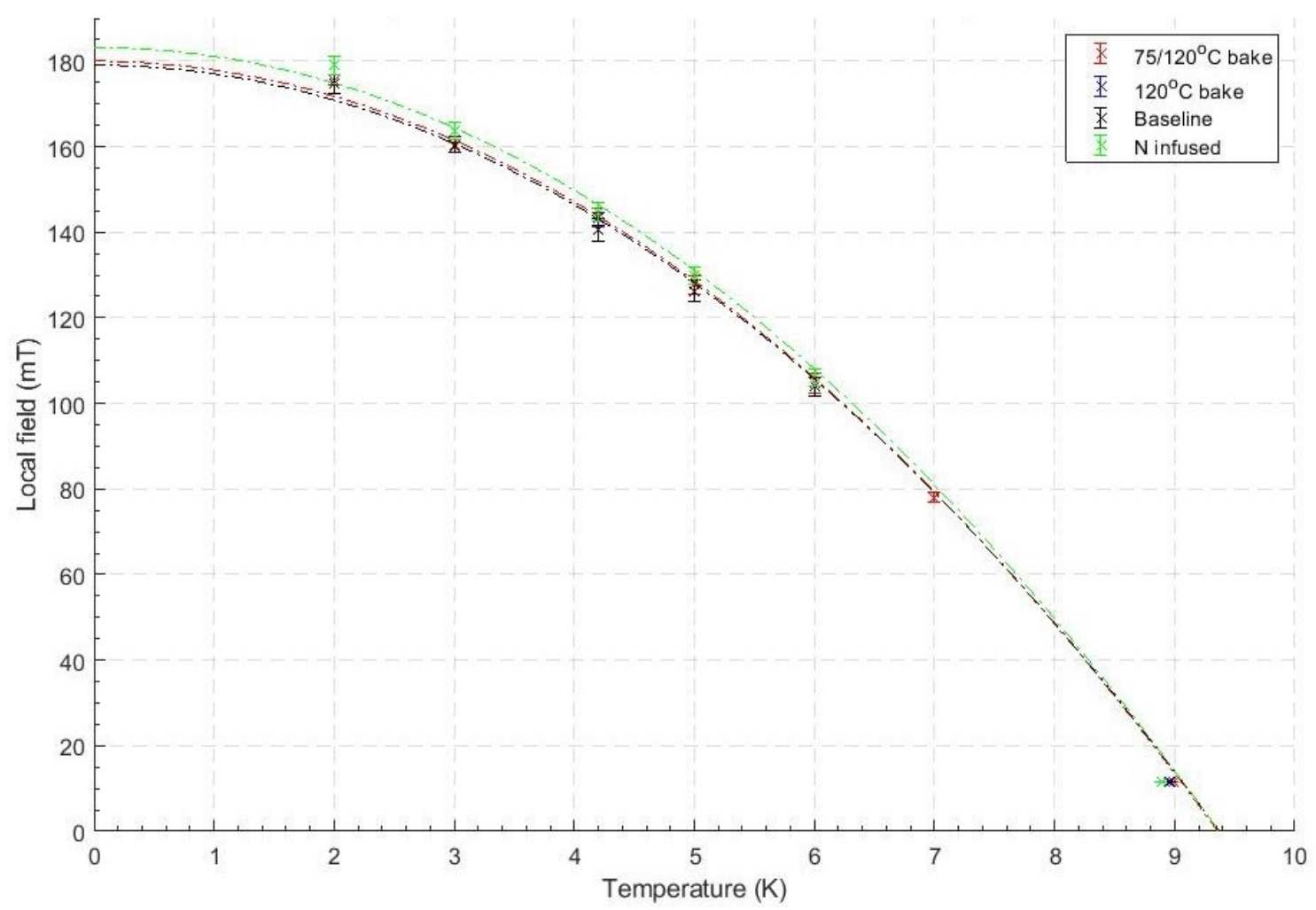

Figure 3. The field of first flux penetration as a function of temperature for all 4 samples. The line of best fit is shown for baseline and $75 / 120^{\circ} \mathrm{C}$.

An interesting difference between the four samples is their pinning strength as can be seen in the inset in Figure 4 and Table 2. The difference in magnetic moment for increasing to decreasing $\mathrm{H}_{e x t}$ is indicative of the pinning strength. A pin free sample would yield zero magnetic moment for $\mathrm{H}_{e x t}=0$ in both cases. The baseline sample has the weakest pinning. The pinning strength for the $120^{\circ} \mathrm{C}$ and $75 / 120^{\circ} \mathrm{C}$ samples is larger and very similar, while nitrogen infusion yields evens stronger pinning.

\begin{tabular}{|c|c|c|c|c|}
\hline & \multicolumn{4}{|c|}{$\mathbf{M}_{i}$, emu for each treatment } \\
\hline T, K & Baseline & $\mathbf{1 2 0}^{\circ} \mathbf{C}$ bake & $\mathbf{7 5 / 1 2 0} \mathbf{C}^{\circ} \mathbf{C}$ & $\mathbf{N}$ infusion \\
\hline 2 & $0.44 \pm 0.016$ & - & $0.82 \pm 0.0076$ & $1.9 \pm 0.0063$ \\
\hline 3 & $0.24 \pm 0.0063$ & - & $0.50 \pm 0.0071$ & $1.7 \pm 0.0085$ \\
\hline 4 & $0.22 \pm 0.0058$ & $0.39 \pm 0.0090$ & $0.2847 \pm 0.013$ & $0.88 \pm 0.0051$ \\
\hline 5 & $0.94 \pm 0.0075$ & - & $0.18 \pm 0.0057$ & $0.64 \pm 0.0056$ \\
\hline 6 & $0.082 \pm 0.0084$ & - & $0.14 \pm 0.0055$ & $0.37 \pm 0.0058$ \\
\hline 7 & $0.076 \pm 0.0058$ & - & $0.067 \pm 0.0066$ & $0.19 \pm 0.0058$ \\
\hline 8 & - & - & $0.035 \pm 0.0055$ & $0.055 \pm 0.0055$ \\
\hline
\end{tabular}

Table 2. Irreversible magnetic moment obtained at $\mu_{0} H_{e x t}=0 \mathrm{mT}$ indicative of the pinning strength.

\section{Discussion}

Four $\mathrm{Nb}$ ellipsoids were machined and then annealed to eliminate pinning within the samples to produced accurate results when tested using DC magnetometry. Three of the four samples saw further LTB treatments. The samples were held at a set temperature before a hysteresis loop was measured, from which $\mathrm{H}_{v p}$ was determined taking the well defined demagnetization factor into account. The $\mathrm{H}_{v p}(\mathrm{~T})$ for each sample is shown in Table 1 and Figure 3. Interpolating results to $0 \mathrm{~K}$ yields $\mu_{0} H_{v p}(0 \mathrm{~K})$ $=178.90 \mathrm{mT}$ for the baseline sample. This is comparable to previous measurements using muon spin rotation ${ }^{2}$ of $\mu_{0} \mathrm{H}_{c 1}=174$ $\mathrm{mT}$ and magnetometry $173.5 \mathrm{mT}^{3}$. No significant $H_{v p}$ increase was observed for all LTB samples. This shows that the LTB processes do not yield a SS barrier for flux penetration at least in the DC case. This is different to results obtained for bilayers 


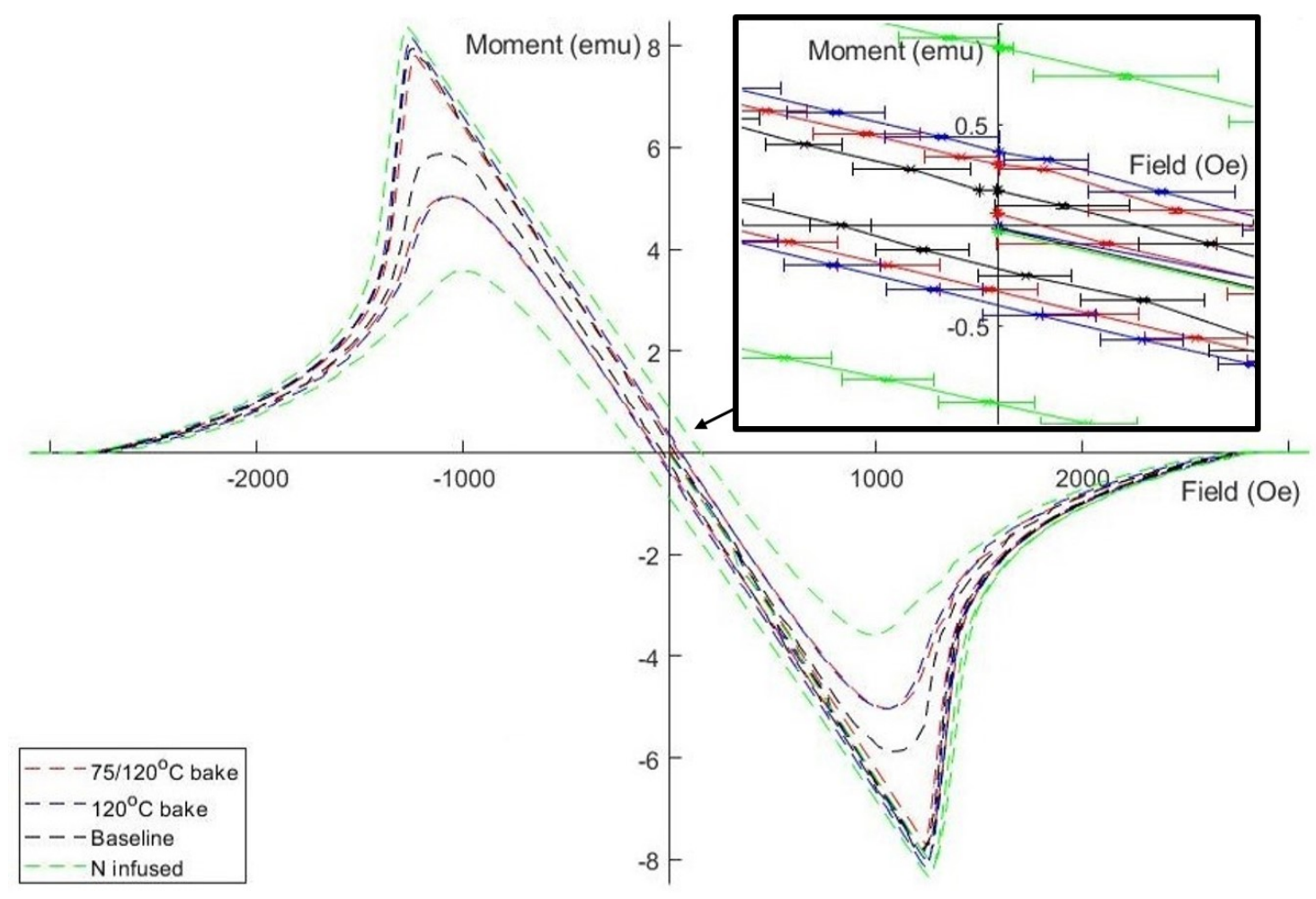

Figure 4. The hysteresis loops at $4.2 \mathrm{~K}$ for all four samples, with a magnified image in the top right for the residual moment when the $\mathrm{H}_{e x t}=0 \mathrm{mT}$.

of $\mathrm{MgB}_{2}$ and $\mathrm{Nb}_{3} \mathrm{Sn}$ on niobium. Tan et $\mathrm{al}^{16}$ found that $200 \mathrm{~nm}$ of $\mathrm{MgB}_{2}$ on $\mathrm{Nb}$ increased the field of first flux penetration by approximately $40 \mathrm{mT}$ compared to uncoated niobium ${ }^{16}$ using a MPMS SQUID magnetometer in a similar experiment to the one presented here. These results are consistent with muon spin rotation ( $\mu \mathrm{SR})$ experiments performed on $\mathrm{MgB}_{2}$ and $\mathrm{Nb}_{3} \mathrm{Sn}$ on niobium samples ${ }^{17}$. These studies suggest an increase in $\mathrm{H}_{v p}$ from a field consistent with $\mathrm{H}_{c 1}$ to a field consistent with $\mathrm{H}_{s h}$ of clean niobium due to the overlayer. These results showed no significant dependence on layer thickness (50-3000nm were tested), therefore suggesting that it is indeed the SS barrier which causes the increase in $\mathrm{H}_{v p}$. This study also found a slight increase of $\mu_{0} \mathrm{H}_{v p}$ from $178 \mathrm{mT}$ to $188 \mathrm{mT}$ for $120 \mathrm{C}$ baked niobium. This effect can potentially be related to surface pinning in a layer thinner the implantation depth of the muons of about $0.15 \mathrm{~mm}$. It should be noted that the effect of the SS barrier in LTB cavities might still be relevant for time-varying RF field. However, the comparison of DC studies on LTB niobium and actual bi-layer samples suggest that this effect is only relevant for actual bi-layers composed of two distinct superconductors.

A measurable effect produced by each treatment is the amount of flux pinning in each sample, shown in Figure 4 . The baseline sample had the least amount of trapped flux, as shown by its magnetic moment, once $\mu_{0} \mathrm{H}_{\text {ext }}$ had returned to $0 \mathrm{mT}$. The low temperature bake samples then had the next greatest moment, and finally the $\mathrm{N}$ infused samples had an even larger moment when $\mu_{0} \mathrm{H}_{\text {ext }}$ had been reduced back to $0 \mathrm{mT}$. Based on this result one can argue that pinning of flux in the outer surface layer is a possible explanation for the delayed high field Q-Slope onset in low temperature. For reference niobium cavities treated by EP have a HFQS onset at $\approx 100 \mathrm{mT}^{18}$. Low temperature $\mathrm{N}$ infusion of a cavity has found to delay the onset of the HFQS until the peak magnetic field on the cavity walls is $\approx 190 \mathrm{mT}^{18}$. It has also been found that subsequent removal of the surface of the cavity by HF rinse returns the high field $\mathrm{Q}$ slope to its previous level, therefore concluding that $\mathrm{N}$ infusion only affects a few nanometers on the surface of the sample ${ }^{18}$. The change in the amount of pinning between all four ellipsoid must be attributed to changes on the surface of the material.

In conclusion the results presented here suggest that the delayed HFQS onset might be due to efficient pinning of penetrating vortices in the outer surface layer. Our measurements and comparison with data on actual bi-layer samples suggest that the SS-boundary between the outer layer affected by LTB and the bulk material does not act as a barrier for vortex penetration. There are other potential mechanisms which are neither supported by or in contradiction to our results. These include reduced RF heating due to a reduction of the surface current and mechanisms which suggest the removal of the cause for the HFQS such as ${ }^{19}$. Further studies should focus on understanding the influence of reduced surface current and pinning on the HFQS. 


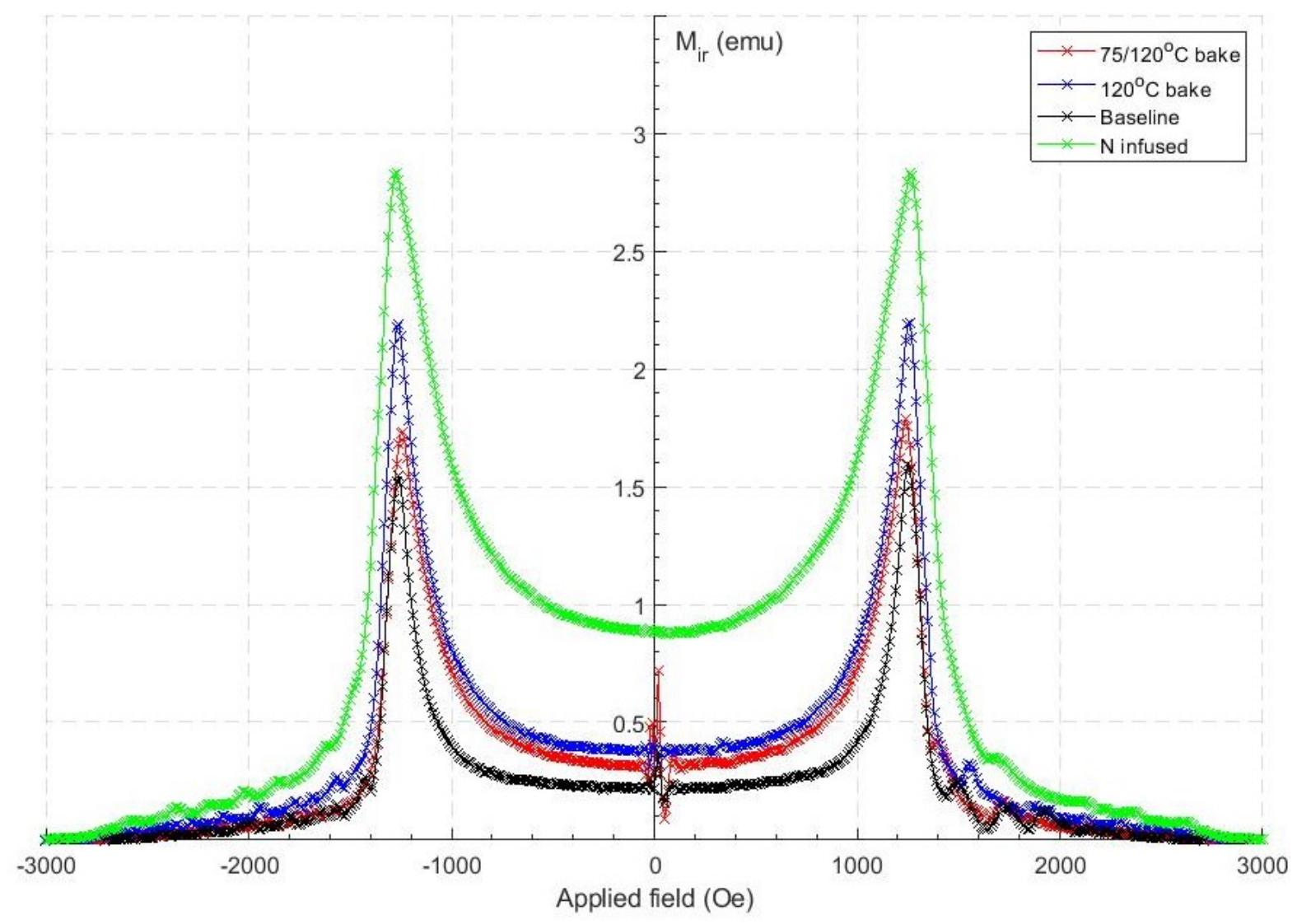

Figure 5. The irreversible pinning $\left(\mathrm{M}_{i r}\right)$ for each treatment at $4.2 \mathrm{~K}$

\section{Methods}

Each ellipsoid was hand polished to remove the edges produced by machining, followed by buffer chemical polishing, BCP, to remove any damaged layers. Following this, the ellipsoids were annealed for 5 hours at $1400^{\circ} \mathrm{C}$ to remove stresses within the $\mathrm{Nb}$ that were present before the machining or produced during machining. This process has shown to remove virtually all pinning $^{2}$. Finally, the ellipsoids had a final round of BCP $(10 \mu \mathrm{m})$ to remove any contaminants that could have been introduced from the oven. One ellipsoid saw no further treatment, and was used as a baseline sample to compare all further treatments too, and is referred to throughout the paper as baseline. One ellipsoid was baked at $120^{\circ} \mathrm{C}$ for $48 \mathrm{~h}$, and another sample was baked at $75^{\circ} \mathrm{C}$ for $4 \mathrm{~h}$ followed by a $120^{\circ} \mathrm{C}$ bake for $44 \mathrm{~h}$ which are referenced throughout the paper as $120^{\circ} \mathrm{C}$ and $75 / 120^{\circ} \mathrm{C}$ respectively. Finally, a sample was sent to FNAL for $\mathrm{N}$ infusion using their standard treatment described in ${ }^{8}$, labelled as the $\mathrm{N}$ infused ellipse.

\section{References}

1. Padamsee, H., Knobloch, J. \& Hays, T. Rf superconductivity for accelerators. New York. A Wiely-Interscience Publ. 129-144 (1998).

2. Junginger, T. et al. Field of first magnetic flux entry and pinning strength of superconductors for rf application measured with muon spin rotation. Phys. Rev. Accel. Beams 21, 032002 (2018).

3. Finnemore, D., Stromberg, T. \& Swenson, C. Superconducting properties of high-purity niobium. Phys. Rev. 149, 231 (1966).

4. Bean, C. \& Livingston, J. Surface barrier in type-ii superconductors. Phys. Rev. Lett. 12, 14 (1964).

5. Transtrum, M. K., Catelani, G. \& Sethna, J. P. Superheating field of superconductors within ginzburg-landau theory. Phys. Rev. B 83, 094505 (2011).

6. Ciovati, G. Effect of low-temperature baking on the radio-frequency properties of niobium superconducting cavities for particle accelerators. J. applied physics 96, 1591-1600 (2004). 
7. Grassellino, A. et al. Accelerating fields up to $49 \mathrm{mv} / \mathrm{m}$ in tesla-shape superconducting rf niobium cavities via $75 \mathrm{c}$ vacuum bake. arXiv preprint arXiv:1806.09824 (2018).

8. Grassellino, A. et al. Unprecedented quality factors at accelerating gradients up to $45 \mathrm{mvm}-1$ in niobium superconducting resonators via low temperature nitrogen infusion. Supercond. Sci. Technol. 30, 094004 (2017).

9. Ciovati, G. Where next with srf. In Paper presented at IPAC2013,Shanghai, China p. 3124 (2013).

10. Romanenko, A. et al. Strong meissner screening change in superconducting radio frequency cavities due to mild baking. Appl. Phys. Lett. 104, 072601 (2014).

11. Kubo, T. Multilayer coating for higher accelerating fields in superconducting radio-frequency cavities: a review of theoretical aspects. Supercond. Sci. Technol. 30, 023001 (2016).

12. Brandt, E. H. Superconductors in realistic geometries: geometric edge barrier versus pinning. Phys. C: Supercond. 332, 99-107 (2000).

13. Quantum Design. Magnetic property measurement system mpms 3 user's manual. Quantum Des. Inc (2016).

14. Wilde, S. et al. Dc magnetometry of niobium thin film superconductors deposited using high power impulse magnetron sputtering. Phys. Rev. Accel. Beams 21, 073101 (2018).

15. Senoussi, S. Review of the critical current densities and magnetic irreversibilities in high tc superconductors. J. de physique III 2, 1041-1257 (1992).

16. Tan, T., Wolak, M. A., Xi, X., Tajima, T. \& Civale, L. Magnesium diboride coated bulk niobium: a new approach to higher acceleration gradient. Sci. reports 6, 35879 (2016).

17. Junginger, T., Wasserman, W. \& Laxdal, R. Superheating in coated niobium. Supercond. Sci. Technol. 30, 125012 (2017).

18. Checchin, M. \& Grassellino, A. High-field q-slope mitigation due to impurity profile in superconducting radio-frequency cavities. arXiv preprint arXiv:2005.05396 (2020).

19. Romanenko, A., Barkov, F., Cooley, L. \& Grassellino, A. Proximity breakdown of hydrides in superconducting niobium cavities. Supercond. Sci. Technol. 26, 035003 (2013).

\section{Acknowledgements}

The authors would like to thank the TRIUMF SRF group and the mechanical workshop for preparing the samples. We acknowledge Martina Martinello for the $\mathrm{N}$ infusion treatment of one of the ellipsoids at Fermi National Laboratory. In addition, we would like to acknowledge Gavin Stenning for providing access to the SQUID magnetometer at Rutherford Appleton Laboratory and providing training on the equipment.

\section{Competing Interests}

The authors declare no competing interests. 